\section{E0328 CORONARY ARTERY CALCIFICATION MAY PREDICT CORONARY HEART DISEASE IN WOMEN PATIENTS}

doi:10.1136/hrt.2010.208967.328

${ }^{1}$ Ling Sun, ${ }^{2}$ Shuzheng Lu. 'Liaoning Fushun Central Hospital; ${ }^{2}$ Beijing Anzhen Hospital

Objectives Using the spiral CT to scan coronary calcification, with qualitative and semi-quantitative method, to predict the presence and extent of coronary artery disease.

Background Early diagnosis of coronary artery disease has been an important clinical issue. Coronary angiography was the gold standard for diagnosis of coronary artery disease, but to have invasive examination, only used in a small fraction of patients. Clinical diagnosis in most patients still need to rely on non-invasive examination. Women have a lower incidence of coronary artery disease, high load test false positive rate. Detection of coronary artery disease in female patients is very important. Coronary artery calcium deposition within the intima is a sign of atherosclerosis. CAC associated with the presence and extent of coronary atherosclerosis.

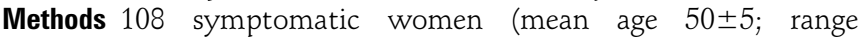
$45-76$ years) received coronary angiography and chest CT scan. CT image shows left main and at least proximal and middle part of anterior descending was considered acceptable CT scans, punctate calcification as mild, segmental calcification as moderate and diffuse as severe. Stenosis were measured in three vessels. Completely normal coronary artery was defined as no stenosis. Stenosis less than $50 \%$ was considered non-obstructive, while more than $50 \%$ stenosis as obstructive coronary artery disease.

Results Of all the 108 patients, 41 confirmed by coronary angiography with normal coronary artery, 67 patients in contrast with the narrow, including 12 non-obstructive, 55 of obstructive stenosis. There were no difference in patient with or without obstructive stenosis. 41 patients with normal coronary angiography showed that 26 were not calcified, 15 with mild calcification. The sensitivity to predict obstructive $\mathrm{CAD}$ was $68.7 \%$, specificity was $92.8 \%$.

Conclusion Symptomatic women patients without CAC on chest CT scan may have less possibility of obstructive CAD. Such patients may not need excessive coronary angiography.

\section{e0329 SAFETY AND FEASIBILITY OF TIROFIBAN IN ELECTIVE PCI OF COMPLEX CORONARY ARTERY DISEASE}

doi:10.1136/hrt.2010.208967.329

Jia Dean, Zhou Yujie, Shi Dongmei, Wang Jianlong, Ge Hailong, Liu Xiaoli. Beijing Anzhen Hospital Affiliated to Capital Medical University

Objectives To observe the effect of tirofiban on cardiac markers, platelet aggregation rate and major adverse cardiac events (MACE) in patients with complex coronary artery disease undergoing elective PCI and discuss the safety and feasibility.

Methods Retrospectively enrolled 676 patients with complex coronary artery disease and divided into conventional treatment $(n=364)$ group and tirofiban $(n=312)$ group. Aspirin and clopidogrel were used in both groups and tirofiban was used in T-group at least $24 \mathrm{~h}$. Observe cardiac markers (Troponin-I and Creatine kinase-MB), platelet aggregation rate and MACE (recurrent angina, revascularization, non-fatal myocardial infarction and cardiac death) at 6-month. Results The baseline risk of the two groups were of no difference. Platelet aggregation rate $(12 \%$ vs $41 \%, \mathrm{p}=0.015)$, TnI $(21 \%$ vs $68 \%$, $\mathrm{p}=0.033)$ and $\mathrm{CK}-\mathrm{MB}(14 \%$ vs $52 \%, \mathrm{p}=0.016)$ at $24 \mathrm{~h}$ after procedure was lower in T-group than that in C-group. Recurrent angina (9.3\% vs $14.3 \%, p=0.046)$ and MACE $(17.3 \%$ vs $23.6 \%, p=0.043)$ at 6-month was lower in T-group than that in C-group. There was no significant difference in revascularization, non-fatal MI and cardiac death. Platelet count was similar in both groups $\left(238 \pm 57 \times 10^{9} / 1\right.$ vs $\left.224 \pm 46 \times 10^{9} / 1, p=0.328\right)$. The minor bleeding events increased in T-group ( $8.2 \%$ vs $3.7 \%, p=0.024)$, there was 1 gastrointestinal bleeding and no intracranial haemorrhage in T-group.

Conclusions Tirofiban can decrease platelet aggregation rate, cardiac markers (TnI, CK-MB) and improve clinical outcomes at 6-month and it is safe and effective to use tirofiban in patients with complex coronary artery disease. These findings indicate that tirofiban is efficacious and safe in complex coronary artery disease patients undergoing elective PCI.

\section{e0330 ONE CASE OF SEVERE TIROFIBAN-INDUCED THROMBOCYTOPAENIA AFTER THE EMERGENCY PCI}

doi:10.1136/hrt.2010.208967.330

Dongyang Xie, Jiumei Cai, Yiming Zhong. Department of Cardiology, Affiliated Hospital of Gannan Medical College, JiangXi Ganzhaou

Clinical Data This was a $73 \mathrm{~kg}$ and 78 years old male patient presented urgently with chest pain for late $3 \mathrm{~h}$ on September 17, 2009. The ECG showed ST segment elevation up to $0.5-0.6 \mathrm{mv}$ in the leads of V1-V4 and the patient was diagnosed as acute anterior myocardial infarction. After admission, urgently checked routine blood test and coagulation function were normal and platelet count was $150 \times 10^{9} / 1$. After treatment with $600 \mathrm{mg}$ oral clopidogrel, $300 \mathrm{mg}$ oral aspirin and intravenous glucoprotein IIb/IIIa receptor antagonist tirofiban in the loading dose of $10 \mu \mathrm{g} / \mathrm{kg}$ and in the continued dose of $0.15 \mu \mathrm{g} / \mathrm{kg} / \mathrm{min}$, the patient was sent to the intervention room. The coronary angiography showed that the proximal part of left anterior descending artery (LAD) was in acute occlusion and percutaneous coronary intervention (PCI) was performed immediately after injected intravenously ordinary heparin sodium $5000 \mu(70 \mathrm{IU} / \mathrm{kg})$. While the guide wire was cross the occlusion part of $\mathrm{LAD}$, the degree of proximal stenosis was up to $95 \%$, a large number of thrombi was existed and the far-end blood flow was TIMI grade 1. After the proximal part of LAD was expanded by nujing plus balloon $(2.0 \mathrm{~mm}$ in diameter, $20 \mathrm{~mm}$ in length), far-end blood flow was TIMI grade 2 and the residual stenosis was $50 \%$. At this time, reperfusion arrhythmias occurred such as frequent premature ventricular contractions and accelerated ventricular rhythm and chest pain aggravated. Three minutes later, the patient recovered sinus rhythm and chest pain significantly alleviated. After implanted Fire bird drug-eluting stent $(3.5 \mathrm{~mm}$ in $\mathrm{dm}, 24 \mathrm{~mm}$ in length), the residual stenosis of LAD was disappeared and the distal blood flow reached TIMI grade 3 . The vital signs were stable during the procedure and the patient was safe back to CCU ward after operation. Low molecular weight heparin sodium were regularly injected subcutaneously in dose of $6000 \mu$ one time every $12 \mathrm{~h}$ and tirofiban were injected continuously by micro pump. The first day after operation, patient had systemic ecchymosis and the platelets count decreased to $3.0 \times 10^{9} / 1$. So the patient was taken out of all anti-platelet and anticoagulant drugs and transfused a unit of person platelet. After rechecking routine blood test, the platelet count increased to $15 \times 10^{9} / 1$ and the ecchymosis did not become heavier. The second day after operation, bone marrow reports said megakaryocyte proliferation activity and too many platelets were destruction. Repeated routine blood test showed that platelet count gradually increased daily. The fifth postoperative day, platelets count was up to $110 \times 10^{9} / 1$ and stabilised at $120-180 \times 10^{9} / 1$ from then on. Skin ecchymosis gradually subsided. So patient was treated with oral clopidogrel in dose of $75 \mathrm{mg}$ daily, oral aspirin in dose of $100 \mathrm{mg}$ daily, low molecular weight heparin sodium in dose of $6000 \mu$ one time every $12 \mathrm{~h}$.

Discussion This was an elderly male patient with acute myocardial infarction. Before emergency PCI operation, the platelet count was normal. At perioperative period, antiplatelet and anticoagulation 